\title{
Attention et conscience : à la croisée de la phénoménologie et des sciences cognitives
}

Natalie Depraz

\section{(2) OpenEdition}

1 Journals

Édition électronique

URL : http://journals.openedition.org/alter/1683

DOI : $10.4000 /$ alter.1683

ISSN : 2558-7927

Éditeur :

Association ALTER, Archives Husserl (CNRS-UMR 8547)

\section{Édition imprimée}

Date de publication : 1 octobre 2010

Pagination : 203-226

ISBN : 2-9522374-6-8

ISSN : $1249-8947$

\section{Référence électronique}

Natalie Depraz, «Attention et conscience : à la croisée de la phénoménologie et des sciences cognitives », Alter [En ligne], 18 | 2010, mis en ligne le 01 juin 2020, consulté le 28 juin 2020. URL http://journals.openedition.org/alter/1683 ; DOl : https://doi.org/10.4000/alter.1683 


\title{
ATTENTION ET CONSCIENCE : À LA CROISÉE DE LA PHÉNOMÉNOLOGIE ET DES SCIENCES COGNITIVES
}

\author{
Natalie Depraz
}

On imagine mal de prime abord que je puisse être attentive sans que la conscience soit d'une manière ou d'une autre au rendez-vous! Je suis en train d'écouter le serveur plaisanter avec les clients, et on ne peut pas dire que je sois «inconsciente » au moment où j'écoute «attentivement » ses plaisanteries! Bien sûr, être conscient ne correspond pas ici à une conscience explicite, réflexive ou interrogatrice, davantage à une conscience en acte, plus minimale, une sorte de présence en ligne de fond à ce que je fais. Une fois cette distinction posée entre ces deux types de conscience, on aura tendance assez spontanément à identifier cette dernière forme de conscience et l'attention.

Pourtant, phénoménologues comme scientifiques de la cognition attirent notre « attention » sur des micro-phénomènes irréductibles à cette équivalence : je suis affectée par les bruits environnants du café où je me trouve, qui détournent mon attention, de façon récurrente, de mon activité d'écriture de ce texte, et cette affection répond à une forme d'attention à la situation qui a le caractère d'un accueil, d'une sorte de disponibilité que Husserl ou James diraient "passive », mais qui relève d'une spontanéité ouverte ; ces affections multiples me traversent et entrecoupent mon activité attentionnelle consciente dirigée (focalisée) sur la production de ce texte. Très clairement, cette attention ouverte n'est pas consciente, ou alors, elle renvoie à une forme très organique et primitive de mode de relation au monde! Au moment où je suis centrée sur l'écriture de mon texte, les bruits autour de moi coexistent mais je $\mathrm{n}^{\prime}$ en ai pas conscience $^{1}$ ! Depuis un peu plus $\mathrm{d}^{\prime}$ une dizaine d'années, on a fait apparaître des cas de cécité neuronale associés à des reconnaissances visuelles (de lettres, de visages) en l'absence, chez le sujet, d'une conscience de cette compétence; c'est ce que l'on nomme la «vision

1. E. Husserl, De la synthèse passive, Grenoble, Millon, 1998, et N. Depraz, pour la mise en pratique des exemples, Lire Husserl en phénoménologue, Paris, PUF/CNED, 2008. 
aveugle » (blindsight), où le sujet, privé de la vision de la partie droite du champ perceptif, est cependant capable de reconnaître sans voir. Ainsi, il y a non-conscience subjective d'une capacité visuelle effective, pourtant déficitaire de façon neuronale (pas d'activation du cortex visuel primaire) ${ }^{2}$.

Ces deux contre-exemples permettent de poser la question de la noncoïncidence/coextensivité entre conscience et attention. Pour construire ce problème, je vais tout d'abord introduire des termes qui ont contribué à la confusion entre l'attention et la conscience, à savoir la réflexion et la perception, de façon à proposer des distinctions plus fines; dans un deuxième temps, je m'intéresserai à la dynamique des deux phénomènes (prendre conscience, devenir attentif), pour leur donner du relief et mieux cerner leur irréductibilité mais aussi leurs lieux possibles de recoupement.

\section{Conscience, attention, perception, réflexion : le problème du pré-réfléchi}

Mon hypothèse dans ce premier moment est de déployer les articulations entre ces quatre termes, que l'on confond souvent, et d'évoquer sur cette base certains arguments critiques, historiques et conceptuels, qui interrogent la pertinence du terme de "pré-réfléchi ». Je vais tout d'abord restituer certains jalons de la construction $\mathrm{du}$ concept $\mathrm{d}^{\prime}$ attention et de son découplage par rapport à la conscience, puis je clarifierai la relation de l'attention à la perception et à la réflexion; ensuite je m'intéresserai aux motifs, internes à la phénoménologie husserlienne, de dissociation précoce de la réflexion et de l'attention; ce qui m'amènera finalement à ré-interroger la pertinence du pré-réfléchi et à proposer à sa place la notion de dynamique attentionnelle.

\section{A. La construction du concept d'attention et son découplage par rapport à la conscience}

Alors que la perspective classique (Descartes puis Leibniz) met au premier plan l'être-conscient comme aperception de soi et voit dans le modèle de la réflexion comme «perception de perception » la formalisation bouclée de cet être-conscient, Husserl au début du XXe siècle s'intéresse davantage à l'avoir-conscience de... comme mouvement de déploiement de soi vers son dehors. Au retour sur soi, par quoi la conscience se replie sur son monde intérieur, le fondateur de la phénoménologie oppose une ouverture du sujet sur lui-même selon un double geste, disposé vers les

2. L. Naccache, Le nouvel inconscient, Paris, Seuil, 2006 ; S. Dehaene \& J.-P. Changeux, « Ongoing spontaneous activity controls access to consciousness: a neuronal model for inattentional blindness ", PLoS Biol., 3, e141 (2005). Plus tôt, le livre inaugural sur lequel je vais revenir dans le deuxième temps : A. Mack \& I. Rock (1998), Inattentional Blindness, Cambridge MA, MIT Press. 
autres et tourné vers le monde. C'est accueillir pleinement l'extériorité, à savoir l'autre que soi, autrement dit, ce qui nous excède, nous transcende, en en ressaisissant au même moment la part d'étrangeté qu'il suppose d'accueillir en moi-même pour pouvoir le recevoir en plénitude comme irréductiblement autre.

Comment se déploie le mouvement initial d'une telle ouverture intentionnelle? On connaît la formule célèbre selon laquelle toute conscience est « conscience de quelque chose ${ }^{3}$ » : elle fournit la définition formelle de cette propriété nouvelle de la conscience qu'est l'intentionnalité. À partir de cette redéfinition de la conscience ${ }^{4}$, on peut à son tour ré-interroger la dynamique de son devenir-conscient, à savoir la genèse du mouvement par lequel le sujet s'aperçoit d'une dimension de lui-même qu'il n'avait tout d'abord pas vue.

C'est cette avancée à partir de Husserl et dans son au-delà possible qui me conduit à passer sur un mode critique d'une conception de la conscience comme intentionnalité à une conception renouvelée de la conscience comme attention. Ce mouvement suppose une refonte parallèle de l'attention, destinée à mettre en défaut sa dimension seulement mentale et focale, bref, abstraite, et à libérer en elle une qualité insigne de "vigilance » (qui inclut éveil, veille et disponibilité, et que je vais ici évoquer un peu trop rapidement). En fait, la relation entre conscience et attention dans leur recompréhension mutuelle en termes de "vigilance » correspond au cadre plus large d'une recherche en cours portant sur la phénoménologie de l'attention. À ce titre, je fais l'hypothèse, à vérifier, selon laquelle l'attention entendue comme vigilance n'est pas une propriété de la conscience (comme pouvaient l'être l'intentionnalité ou le temps vécu); c'est la conscience elle-même qui devient une propriété (parmi d'autres) de la vigilance attentionnelle, comme l'éveil et la disponibilité. Cependant, à ce stade, une telle hypothèse demeure abstraite, et doit, pour être vérifiée, être non seulement argumentée dialectiquement ${ }^{5}$, mais éprouvée sur un mode expérientiel et pragmatique, ce que, encore une fois, je ne ferai pas ici.

Pour amorcer la construction de cette relation délicate entre conscience et attention, on peut faire trois remarques préliminaires, propres à cerner trois propriétés structurelles de l'attention, qui viennent compléter et prolonger la propriété nommée initialement, à savoir la conscience, ainsi que celles qui concernent davantage le caractère processuel de la vigilance (éveil, veille et disponibilité) : l'une concerne la propriété de l' «intimité », par distinction $\mathrm{d}^{\prime}$ avec le comportement et en lien avec une approche en première personne (sans qu'il s'agisse pour autant d'un « privé » anecdotique) ; l'autre a trait au

3. E. Husserl, Méditations cartésiennes, Paris, PUF, 1994, §14, p. 78.

4. Pour plus de détails, cf. N. Depraz, La conscience. Approches croisées des Classiques aux sciences cognitives, Paris, A. Colin, 1999, p. 69-70 et p. 71-79.

5. À propos de ce renversement proprement dialectique des relations entre conscience et intentionnalité, cf. R. Barbaras, Introduction à une phénoménologie de la vie, Paris, Vrin, 2008 (dans sa perspective, au profit de la vie). 


\section{L'attention}

caractère transversal et interdisciplinaire de l'attention, à savoir relationnel, ici, social et éthique (par contraste avec son arrimage seulement individuel), et que mettent en scène les méthodes en deuxième et en secondes personnes; la troisième touche à sa caractéristique pratique (par différence avec l'acte avec lequel on le confond souvent, la réflexion, tendanciellement théorisante).

Ces trois propriétés ont pour vertu de ne pas être seulement complémentaires, elles s'auto-contraignent les unes les autres: (1) les travaux introspectionnistes du début du siècle (Stumpf, James, Külpe, Titchener, parmi d'autres) attestent très tôt, quoique sur un mode évocatif (non démonstratif), que les actes internes d'attention sélective ne sont pas nécessairement accompagnés par des signes extérieurs explicites comme les mouvements oculaires ou les changements de position. En fait, c'est Helmholtz, l'un des pères fondateurs de la psychologie expérimentale, qui a très tôt fait apparaître ce découplage entre l'attention visuelle et le positionnement des yeux ${ }^{6}$. En ce sens, l'attention est un thème qui a dès le départ mis mal à l'aise les behavioristes et a en revanche remarquablement intéressé les promoteurs des actes internes ${ }^{7}$. (2) Souvent considérée comme coextensive à la conscience, l'attention se trouve en réalité à la croisée de plusieurs disciplines (phénoménologie, psychologie, neurobiologie, psychiatrie, etc.) qui découvrent aujourd'hui des lieux d'articulation, des points de passage, des zones de continuité entre elles. Elle possède de fait une portée relationnelle et trans-individuelle, indissolublement sociale, éthique voire politique et économique. (3) Le phénomène de l'attention, contrairement à la conscience, contient en lui-même une dimension méthodologique inhérente à sa phénoménalité (être attentif recèle sa propre attention immanente à l'attention, sans surenchère réflexive ni spéculative), ce qui permet d'éviter de dissocier totalement, de façon naïvement duelle, description, pratique, exploration d'une part, réflexion sur la description, savoir de la pratique, thématisation de l'exploration d'autre part. Il offre ainsi, de façon intrinsèque, phénoménale, une continuité entre l'objet et la méthode, là où la relation entre objet et méthode est la croix de nombreux débats sur le lien entre la théorie et la pratique.

Ce découplage entre attention et conscience (articulé à partir des propriétés phénoménologiques susdites : 1) intimité ; 2) relation ; 3) pratique) permet de se situer dans un débat dominant aujourd'hui en sciences cognitives : contrairement à une option assez répandue, qui identifie purement et simplement conscience et attention ou, du moins, fait apparaître, mais sans la décrire précisément, leur relation étroite ${ }^{8}$ et, tout autant, contrairement

6. J.-P. Mialet, L'attention, Paris, PUF, 1999, p. 18.

7. H.E. Paschler, The Psychology of Attention, MIT Press, 1999, p. 6.

8. I. Rock \& D. Gutman (1981), "The effect of inattention on form perception", Journal of Experimental Psychology: Human Perception and Performance, 7, p. 275-285; A. Mack \& I. Rock (1998), Inattentional Blindness, Cambridge MA, MIT Press; H.H. Gander, «On attention», Research in Phenomenology, 2007. 
à l'interprétation qui consiste seulement à montrer, plus dynamiquement, comment l'attention émerge d'un apprentissage implicite inconscient (implicit learning $^{9}$ ), je voudrais faire apparaître en quoi l'expérience attentionnelle prise dans toute son amplitude conduit en fait à redimensionner radicalement la conscience elle-même. Celle-ci n'est plus seulement réflexive ni simplement intentionnelle mais proprement "attentionnelle", ce qui conduit par là même, on l'a dit, à une transformation corrélative de la notion classique d'attention, elle-même réformée en « vigilance ».

\section{B. De la difficulté à identifier l'attention à sa marginalisation par rapport à la} perception et à la réflexion : des Recherches logiques à Philosophie première

Dans ce qui précède, j’ai évoqué en passant, moyennant la désarticulation entre conscience et attention et l'amorce de sa ré-articulation avec le concept de vigilance, l'ambivalence dans laquelle se trouve l'attention vis-à-vis de la perception et de la réflexion, ainsi que la nécessité de libérer la première de ses deux acolytes historiques. Il convient à présent de revenir au contexte initial de la naissance de l'attention, pour mieux comprendre comment celle-ci a pu, chez Husserl encore, rester par moments dépendante des expériences perceptive et réflexive. Un tel examen historique est décisif, car il permet d'identifier les lieux prégnants de cristallisation entre l'attention et le modèle gnoséologique (objectif, individuel, théorique) de la perception/réflexion, c'est-à-dire, par là même, de comprendre comment une telle identification peut être soutenue aujourd'hui, mais aussi à partir de quels arguments il est possible de se décaler et de faire droit à une autre entente de l'attention (intime, relationnelle et pratique). Dans l'espace-temps qui m'est ici imparti, je me contenterai ici de donner quelques éléments de cette problématique historique.

C'est le spectre du «psychologisme », c'est bien connu, qui motive dès les Recherches logiques, dans les «Prolégomènes à la logique pure » tout d'abord, la légitimité exclusivement rationnelle de l'attention et la revendication sans reste de l'attention comme attention réflexive. À cet égard, Husserl s'inscrit dans un cadre général où l'attention est considérée comme l'activité d'un sujet rationnel connaissant et la réflexion l'aptitude du sujet rationnel à se connaître. Un tel mode de conscience du sujet est caractérisé par la rationalité de ce dernier, sa visée principale dans sa relation au monde (aux objets) étant la connaissance. Depuis ce cadre de la connaissance rationnelle, attention et réflexion sont liées par un lien de fondation logique en raison: 1) l'attention est un acte de connaissance de l'objet où joue en basse

9. Cf. T. Lambert, «Visual orienting, learning and conscious awareness » and Y. Jiang et M.M. Chun, "Contextual Cueing. Reciprocal influences between attention and implicit learning ", in : Attention and Implicit Learning, éd. L. Jimenez, Amsterdam, Benjamins Press, 1998, Advances in Consciousness Research, respectivement p. 253-275 et p. 277-297, notamment p. 278. 


\section{L'attention}

continue, sur un mode indivis voire confondu, la perception de cet objet (une saisie enracinée dans la sensation mais s'en libérant, s'en abstrayant, car les sens sont générateurs d'illusion perceptive: trompeurs), et cette perception est alors d'emblée appréhendée comme un acte intellectuel où je suis amené à juger que ce que je vois est vrai ; l'attention est alors recouverte sous la perception jugeante, et celle-ci me conduit à affirmer, à poser l'êtrevrai de l'objet, à partir de quoi une connaissance possible en procède. Cette problématique trouve directement ses racines dans la conception cartésienne, sur laquelle je ne reviendrai pas ici.

La réflexion, quant à elle, est un acte de connaissance de soi pris comme objet, elle-même fondée sur l'attention perceptive entendue comme acte de connaissance pour ainsi dire direct de l'objet. Cette structure de fondation place l'attention en position tout à la fois motrice (mais non relevée comme telle) et subordonnée : l'attention à l'objet sur fond d'activité perceptive permet la réflexion sur soi, mais, tout autant, la réflexion est un acte qui opère un retour sur l'intériorité du sujet que ne permet pas l'attention, limitée à la visée connaissante de l'objet externe. Formulé autrement, la distinction entre perception et réflexion répond à une dichotomie claire, la première se construisant comme une visée focalisatrice de l'objet qui articule la modalité sensorielle à l'identification formalisante de l'objet en donnant lieu à la connaissance, alors que la seconde engage un détournement par rapport à l'objet et un retournement sur le soi ; l'attention introduit en revanche un brouillage qui renvoie à la difficulté à lui assigner une place claire face à ce bel ordonnancement qui régit la différence entre la conscience de l'objet (perception) et la conscience de soi (la réflexion). En effet, en lien avec la perception, l'attention s'inscrit dans une visée de focalisation sur un objet, mais, par contraste avec elle, elle tient davantage à un mode de présence à l'objet, à une "tenue", à un "rehaussement", et se libère ce faisant du contenu visé, du résultat objectif. Par ailleurs, l'attention ne joue pas seulement un rôle dans la relation à l'objet : en tant qu'acte vécu, elle témoigne d'un mouvement auto-centré de l'esprit, et engage ainsi, comme la réflexion, le soi, sans impliquer pour autant un mouvement de retour sur soi, ni un redoublement de la perception.

Bref, la construction rationnelle de la connaissance pouvait sembler, de prime abord, impartir à chaque acte des fonctions différenciées: l'objet externe pour l'attention, l'état interne pour la réflexion. En fait, une telle délimitation révèle ses limites : d'une part, la dichotomie intérieur/extérieur trahit une limitation de l'attention à l'objectivité directe, alors que l'attention porte aussi sur le soi, et décrit un espace de relation du sujet à lui-même non reconductible à la relation à l'objet externe et tout à la fois irréductible au retour réflexif sur soi ; d'autre part, elle trahit une omnipotence de l'activité réflexive du sujet dans le champ de l'activité interne, comme si l'expérience du soi ne pouvait pas passer par un autre acte ou vécu que par la réflexion, à savoir par une activité de second degré, que l'on décrit parfois, on l'a dit, comme un redoublement de la perception, problématique qui aura été 
largement développée par Leibniz. Je n'insiste pas ici sur ce point. Une telle distinction renvoie à une appréhension insatisfaisante de l'activité réflexive elle-même, laquelle ne saurait, pas plus, être une simple structure de répétition de l'activité perceptive, de l'extérieur à l'intérieur. Si l'on considère la réflexion comme un tel emboîtement de perceptions, on aboutit très vite à une possible "régression à l'infini », qui conduit à décoller de l'expérience et à rentrer dans une spéculation qui tourne à vide. Une telle enflure sera au centre de la problématique de Fichte, pour qui l'attention est réflexive ou n'est pas: on y a affaire à un renversement de la théorie classique de la connaissance, où l'attention à l'objet restait le fondement de la réflexion du soi sur lui-même. La radicalisation fichtéenne produit une inversion des conditions: l'attention ne peut être que réflexive, et la réflexion (conscience de soi) devient alors la condition de la conscience de l'objet (de la perception).

La tradition philosophique de Descartes à Fichte construit un concept d'attention qui s'inscrit dans le cadre de la connaissance de l'objet et suppose, pour ce faire, un sujet caractérisé par sa maîtrise interne (volonté) et son contrôle de l'objet (raison). En ce sens, être attentif, c'est être centré sur soi et se focaliser sur l'objet pour en identifier les propriétés. Husserl s'inscrit pour une part dans cet héritage rationaliste et idéaliste de valorisation de la philosophie réflexive et d'une perception d'emblée logicisée. Le fondateur de la phénoménologie hérite de cette entente rationnelle de la philosophie, visible à travers le primat qu'il accorde à la logique. Aussi la critique de la logique psychologique va-t-elle de pair avec la mise en question d'une conception empiriste de la théorie de la connaissance qui considère les lois de la pensée comme des lois naturelles ${ }^{10}$. Depuis cette critique, l'attention est rejetée, n'est même pas thématisée par Husserl ici, au nom de sa mise en cause de l'approche psychologique empiriste ; seule la structure de la réflexion trouve grâce à ses yeux, car elle seule est compatible avec une logique pure qui met en avant l'idéalité du perçu et, à terme, dans la période ultérieure, à partir de 1913, l'idéalisme transcendantal de la phénoménologie.

D'ailleurs, on peut trouver chez Husserl lui-même des arguments qui valident une telle approche réflexive de l'attention: dans la cinquième Recherche logique, il déploie une théorie de la conscience qui produit une alliance entre l'acte réflexif et le vécu attentionnel, au titre de leur mise en relief commune de l'unité immanente du soi, identité qui traverse les actes et les vécus en y restant impliqué. C'est cette même vision de l'unité dynamique du soi participant et vivant dans ses actes et ses vécus qui conduira Husserl, dans Philosophie première II, en 1923-24, à tenir ensemble la configuration expérientielle propre à la voie de la psychologie entre attention, réflexion et réduction, ces trois modes de conscience (vécu, acte, méthode) œuvrant de concert à la mise au jour de la vie unificatrice de la

10. Op. cit., chapitre IV, § 22 ; chapitre VIII, § 41, p. 171 ; § 44, p. 185 ; 449 , p. 199. 


\section{L'attention}

conscience par la présence de son soi immanent. Dans son effort de systématisation de la théorie de la réduction, Husserl est ainsi amené à passer à nouveau à la trappe l'attention au profit de son achèvement réflexif puis réductif. Derechef, le vécu attentionnel demeure masqué et, au mieux, subordonné ici à la visée réflexive et réductive.

Qu'en est-il de ce sens dit "empirique »de l'attention qu'aura critiqué Husserl ? Son argument à l'égard des théories empiristes dans la 2e Recherche logique (Locke et Hume d'ailleurs, notons-le, pas Condillac) repose sur leur conception atomiste de l'objet, ce qui attribue à l'attention la fonction d'abstraire, au sens littéral de séparer, de détacher du contexte, de se focaliser exclusivement sur une partie discrète qui devient son objet. Bref, objet et acte sont façonnés l'un par l'autre: au pouvoir d'abstraction de l'attention répond un objet construit comme élément isolé ${ }^{11}$. Dès lors, une telle «discrétisation » de l'objet requiert une reconstruction de son identité au-delà de sa réalité factuelle individuelle, ce qui conduit à faire droit à l'association, puis à la généralisation entendue comme identification du même ${ }^{12}$. En identifiant ainsi les «limites de la théorie abstractive de l'attention du point de vue de la phénoménologie ${ }^{13}$ », à savoir la nondistinction entre l'objet (contenu psychique) et l'acte, l'identification par Locke de l'objet d'attention à un contenu psychique, ${ }^{14}$ et la nondifférenciation des formes mêmes d'abstraction (sensible/non sensible), ${ }^{15}$ Husserl ouvre la voie à un sens authentique de $l^{\prime}$ attention, ${ }^{16}$ qui se situe à un niveau d'amplitude tel qu'il embrasse la distinction intuition/signification. C'est dire que l'attention englobe la pensée, pas seulement l'intuition, et que les actes d'attention peuvent avoir des objets multiples, contenu sensible, psychique, mais aussi formel, comme dans le cas des actes de visée de signification. C'est dans ce contexte extensif que l'on voit apparaître la première occurrence d'une acception de l'attention qui va devenir centrale en 1904-1905 et après, à savoir la «préférence», ou encore «l'acte de remarquer ${ }^{17}$ ». Il s'y agit d'amener à la conscience un contenu, de le détacher en l'éclairant et en le fixant.

Mais dans la 5e Recherche logique, qui présente la phénoménologie comme une psychologie descriptive des vécus, Husserl ré-identifie les parts respectives de la conscience et de l'attention au profit de la première ; d'une part, il s'agit d'examiner la «plurivocité du terme de conscience », 18 d'autre

11. Recherches logiques, Paris, PUF, vol. 2, deuxième recherche, chapitre III, « Abstraction et attention ", $\$ 13$ : "Théories nominalistes qui conçoivent l'abstraction comme l'œuvre de l'attention ".

12. Op. cit., $\S 18:$ : La théorie de l'attention comme pouvoir de généralisation ».

13. Op. cit., $\$ 22$.

14. Op. cit., p. 187-188.

15. Op. cit., p. 190.

16. Op. cit., § 22-23, p. 192-194.

17. Op. cit., p. 192.

18. Op. cit., chapitre 1 : la conscience, $\S 1$, p. 144-146. 
part, ce qu'il en est de « la fonction de l'attention ${ }^{19}$ ». Sans pouvoir entrer ici dans l'analyse détaillée de ces paragraphes, ce qui ressort, c'est le rôle crucial mais aussi compliqué que Husserl accorde à la conscience, en en distinguant trois sens principaux, en en montrant la complexité en relation avec la thématique du moi, du vécu, de l'acte psychique, alors que l'attention se voit réduite, en un paragraphe, à un rôle de marqueur, dans le cadre d'un exemple d'acte complexe concernant la distinction entre le mot et le sens. On voit réémerger ici les termes de «préférence », mais aussi celui d' « influence » en tant que traits caractéristiques de la fonction de l'attention: ce «fait général » (de l'attention) n'a pas été « suffisamment élucidé », écrit Husserl, mais il ne prétend pas ici « développer» une « "théorie" de l'attention ». On ne saurait être plus clair.

\section{La dissociation précoce de la réflexion et de l'attention : du cours de 1904-1905 aux textes sur la synthèse passive}

Ce qui ressort des deux points précédents, c'est le poids de l'acte réflexif à titre de composante définitionnelle de la conscience, vis-à-vis de quoi l'attention est le plus souvent écartée en vertu de son marquage essentiellement empiriste et seulement évoquée dans son statut fonctionnel, ainsi que le rôle basique de l'acte perceptif comme basse continue où se peut venir se greffer à titre adjacent l'acte attentionnel. Tel est semble-t-il le paysage standard des relations entre réflexion, perception et attention dans la phénoménologie de Husserl. Est-ce son premier et son dernier mot?

Je voudrais montrer qu'il y a davantage chez Husserl, y compris très précocement, à savoir dans les années autour des Recherches logiques, que cette présence critique ou latérale de l'attention. C'est le contexte d'une confrontation serrée avec son maître Carl Stumpf à propos de la conception de l'attention de ce dernier comme « plaisir à remarquer » qui va attirer plus avant l'attention du phénoménologue sur la singularité de ce vécu et le conduit à ménager un contraste net avec la perception et, plus encore, avec la réflexion ${ }^{20}$. A cet égard, un passage d'un texte intitulé «Directions de l'attention ", qui a émergé dans le contexte de cette discussion, est particulièrement éloquent: «(...) la conscience dans laquelle se constitue objectivement le représenté en tant que tel est un acte au second degré, une réflexion. La représentation directe lui est sous-jacente, celle du papier. Je suis dirigé vers le papier. J'opère alors la réflexion. Je ne vis plus dans la direction vers le papier, je ne l'accomplis plus de manière vivante, il se produit donc une modification, par quoi la vie s'enfuit, quoique la direction demeure "maintenue". Et puis, dans une nouvelle représentation et une nouvelle

19. Op. cit., chapitre $2, \S 19$, extrait p. 215-217.

20. E. Husserl, Hua XXXVIII, Wahrnehmung und Aufmerksamkeit (T. Vongehr et R. Giuliani éd.), Dordrecht, Kluwer, 2004, traduction française par N. Depraz sous le titre Phénoménologie de l'attention, Paris, Vrin, 2009. L'Introduction retrace notamment le détail de la discussion avec Stumpf. 


\section{L'attention}

position, une direction actuelle se porte vers les événements de l'acte qui s'est fait objet et caput mortuum par la réflexion, vers ce qui part du moi, vers l'orientation en direction de ce qui est orienté en tant que tel, vers son caractère etc., et l'objectivation actuelle possède à nouveau des composantes de représentation et un caractère.

Mais le plus important, c'est de prendre garde au fait que la réflexion dans laquelle la visée modifiée en tant que telle est saisie, n'est pas une simple "conversion du regard" de "l'aperception" comme serait ce rayon de l'orientation qui est présent dans l'acte "vivant" ([dans] l'acte accompli, en l'occurrence, en cela, dans la "représentation" vivante de ce qui est "objectif" à quoi se "rapporte" l'acte), et lui confère précisément une orientation vers ce qui est "objectif" pour lui. L'acte vivant, spontané, tel qu'il est, peut seulement avoir cette orientation et, en tant qu'acte de prise de position, ce qui est "objectif" vers quoi il s'oriente est cela même par rapport à quoi il prend position. Dès que la réflexion idéatrice portant sur ce qui est visé entre en scène, l'acte se modifie. Cela signifie qu' "il" est sous-jacent à titre de présupposition, mais pas sur un mode non modifié, plutôt [sur] un mode modifié. Il n'est plus l'acte originellement accompli, mais un accomplissement mort. Le rayon vivant de l'attention s'est retiré de "lui", la direction-vers se porte à présent ailleurs, et cela signifie qu'il y a là un nouvel acte, et l'ancienne direction-vers est devenu quelque chose de modifié sur un mode non-vivant. Et il en va de même pour la prise de position. Nous avons à présent une nouvelle prise de position vivante, et l'ancienne est non modifiée en tant que non vivante.

Si nous entendons par "conversion attentionnelle" spontanée la spontanéité de la direction-vers et de la prise de position, il y a dans la réflexion un mouvement de détournement, pour autant que la conversion attentionnelle vaut pour quelque chose de nouveau, et le discours du détournement réflexif se justifie dans la mesure où tout ce type de vitalité du détournement possède le caractère d'un rayonnement à partir du point-source de la vie, à partir du "moi pur", porteur de toute vitalité21."

J'ai voulu livrer ici cet extrait in extenso, car il est véritablement exemplaire de la distinction des deux actes que sont la réflexion et l'attention, distinction qui s'énonce ici très clairement en termes de gestes kinesthésiques différents : l'attention est un mouvement global par lequel je me tourne (wende... zu) tout entier, corps et esprit, me dirige (richte) et m'oriente (orientiere) sur un mode que Husserl qualifie de "vivant », parce qu'il est «spontané » et «non-modifié » : il s'agit en bref d'un vécu dont la vitalité demeure entière parce qu'il ne s'objective pas en un acte de saisie identifiante et idéatrice de l'objet, lequel caractérise en revanche la réflexion. Celle-ci, en effet, n'est plus « conversion » (Zuwendung), mais « détournement » (Abwendung) et « retournement » (Umwendung). Bref, l'attention est ouverture vivante et vitale, la réflexion, renfermement sur soi enfermant et mortifère :

21. Ниа XXXVIII, n4, c), p. 389-390. 
caput mortuum. Sous la solidarité sémantique et kinesthésique des deux motifs (tourner : wenden) se joue - Husserl n'aura jamais été plus clair l'axiologie imagée de la vie et de la mort, de l'ouvert et du clos, du spontané et du modifié, ou encore de la prise de position dynamique/génétique et de la représentation statique. Ainsi, la réflexion a partie liée avec l'idéalité judicative, tandis que l'attention est le point-source de la vie. Mais on peut aussi noter, au-delà de l'axiologie post-romantique qui signe cette distinction entre attention et réflexion en termes de vie et de mort, la différence descriptive des gestes kinesthésiques: le vécu attentionnel est décrit comme un mouvement où le corps et la conscience s'accompagnent l'un l'autre en un geste rotationnel unitaire d'ouverture orientatrice ; le vécu réflexif répond au contraire à un geste moteur de rotation inverse qui est repliement et clôture. Pour illustrer cette distinction opératoire, je prendrai un exemple, que j'ai pu expérimenter personnellement, mais qui n'est ici bien sûr qu'indicatif (non explicatif) et demanderait une exploration empirique méthodique «en première personne ${ }^{22}$ », y compris pour en vérifier des inversions expérientielles possibles : il concerne la mobilité des yeux et, plus largement, du visage. Mon attention à la parole d'autrui se manifeste spontanément dans l'ouverture, la vitalité, le frémissement de mon regard dirigé vers l'autre: je suis tout à l'écoute de la situation d'urgence psychiatrique que me raconte mon compagnon, je suis (dira la langue française) «suspendue à ses lèvres » et je vibre à sa parole, réagissant par des mouvements expressifs d'acquiescement (légère ouverture de la bouche) ou de non-compréhension (froncement des sourcils); le passage à la réflexion, l'instant d'après, entraîne en revanche une forme d'absence à la relation immédiate avec lui, une forme d'immobilité de l'expression du regard, de figement des traits du visage, qu'il va interpréter comme une rupture de la relation, une absence voire une indifférence; or, au même moment, je connais une intensité extrême de présence à moi-même, mais celle-ci renvoie à un mouvement interne non visible qui se traduit par une immobilisation externe.

Si l'on reprend à présent le fil historique de l'analyse phénoménologique, on voit comment cette distinction évolue et se trouve reprise dans la période génétique et, notamment, dans les textes consacrés à la synthèse passive : la conversion attentionnelle émerge d'une affection du sujet par l'objet, lequel amorce la dynamique du devenir-conscient et engendre un éveil du sujet à lui-même. Le motif de l'ouverture traverse ainsi le temps pour décrire le processus attentionnel, qu'il se nomme vitalité et orientation comme en 1904-1905, ou affection, éveil et réceptivité comme en 1918-1926 ; en tout état de cause, la dissociation entre le vécu attentionnel et l'acte réflexif est telle

22. Exemple d'une telle exploration méthodique «en première personne » sur un exemple spécifié: N. Depraz, "The "failing' of meaning. A few steps into a "first-person' phenomenological practice ", in Cl. Petitmengin ed. Ten years of viewing from within : the Legacy of F.J. Varela, JCS, 2009. 


\section{L'attention}

dans les années 1920 qu'elle conduit à une distribution textuelle différente. Ce dernier est détaillé dans le cours de 1923-1924, Philosophie première II, notamment dans la quatrième section, où la structure de la réflexivité de la conscience se présente comme l'accomplissement de l'acte du souvenir et de celui de l'imagination, autant de structures de dédoublement du moi aussi nommées scission égoïque (Ichspaltung), qui identifient les différents modes $\mathrm{d}$ 'altérité à soi de l'ego à l'œuvre dans la voie de la psychologie ${ }^{23}$. Bref, on a affaire à deux formes bien distinctes de conscience : 1'une, dans le cadre de la genèse affective de la conversion attentionnelle, est un avoir-conscience affectif en lien avec une ouverture réceptive passive, l'autre, dans le contexte de la voie égoïque de la psychologie, est une conscience réflexive qui enclenche un mouvement de saisie et d'identification de l'objet. À la lumière de l'état de la question dans les années 1920, il est clair que l'attention répond à la première forme de conscience et la réflexion à la seconde.

Pourtant, on a vu que Husserl ne tient pas toujours clairement une telle opposition, qui répond aux attendus de la réforme génétique de la phénoménologie depuis leur réinvestissement transcendantal interne de motifs empiriques issus de la confrontation précoce avec Stumpf. D’ailleurs, de son côté, le critique exemplaire de la philosophie réflexive qu'est MerleauPonty dans la Phénoménologie de la perception, s'il contribue à valider notre suspicion concernant la pertinence de l'acte réflexif (en vertu de son caractère de surplomb et de déformation du vécu), ne rend pas pour autant justice au vécu attentionnel, puisque celui-ci sera simplement mis de côté en vertu de ses défauts solidairement empiristes ou rationalistes, en tant que fonction abstractive et impersonnelle : à sa place, Merleau-Ponty propose de faire droit à l'expérience irréfléchie et pré-réfléchie, ce qui à mon sens nous fait simplement reculer d'un cran, et ce, sans bénéfice, à savoir sans investissement descriptif, l'expérience réflexive restant figée dans sa difficulté structurelle propre ${ }^{24}$.

\section{Du pré-réfléchi, concept négatif, vide et confus, à l'idée de dynamique attentionnelle}

La caractérisation par Merleau-Ponty du mode de conscience situé entre la conscience de soi (dite réflexive) et la conscience automatique (dite anonyme) en termes de conscience "pré-réfléchie » est source de confusion. Certes, elle a le mérite de tenter de différencier et de nuancer, selon une méthode récurrente chez cet auteur, la dualité réflexion/anonymat, mais, pas

23. Cf. à ce propos N. Depraz, Transcendance et incarnation. L'intersubjectivité comme altérité à soi chez E. Husserl, op. cit., chapitre V.

24. À ce propos, D. Zahavi (in Subjectivity and Selfhood, MIT Press, 2005, chapitre 4, p. 73-98), qui ne propose rien de plus dans un chapitre annonçant pourtant une analyse des relations entre "reflection and attention", mais qui se contente de la critique éculée de la réflexion comme déformation et de la proposition finale de faire s'équivaloir, sans plus, attention et pré-réflexion. Cf. mon compte-rendu du livre dans le numéro précédent d'Alter. 
plus que la première topique freudienne, qui introduit entre la conscience et l'inconscient un "pré-conscient », elle ne rend compte du processus et de la dynamique de la conscience; elle ne nous fournit que des états isolés, abstraits, et prétend, en livrant un intermédiaire (le pré-réfléchi), combler un vide expérientiel, alors qu'elle ne propose qu'un prêt-à-penser, un résultat qui ne fait que désigner un problème sans réellement décrire le mécanisme en jeu. Dire en effet que ce mode de conscience est pré-réfléchi, c'est seulement nommer un état antérieur à la réflexion, c'est désigner un état qui précède l'acte réflexif en le nommant de surcroît dans les termes de la réflexion comme s'il en relevait, comme s'il allait de soi qu'il en a la forme et la structure, alors que rien, avant examen expérientiel méthodique, ne peut nous permettre de l'affirmer. Il y a là une présupposition majeure, qui demande un examen critique et empirique approfondi ${ }^{25}$.

En lieu et place de cette coquille vide qu'est le mot-valise de "préréfléchi », nous avons présenté ailleurs ${ }^{26}$ la description structurée et articulée de la dynamique attentionnelle qui répond au processus d'un devenir-conscient plutôt qu'à un état délimité et identifié au sein de ce processus, comme le nomme, dans le meilleur des cas, le terme «préréfléchi ». Le mécanisme de ce processus que nous avons appelé, en usant d'une expression verbale processuelle : «on becoming aware », se compose de façon nodale de trois mouvements internes qui opèrent ensemble : 1) la suspension, 2) la redirection et 3) l'accueil. Nous avons nommé ce foyer du mécanisme l'épochè (en reprenant le terme même de la méthode de Husserl, et en lui conférant une teneur pratique et expérientielle), et désigné ces trois mouvements en termes dynamiques de "phases »: la première a le sens d'une suspension préjudicielle, possibilité même de tout changement dans le type d'attention que le sujet prête à son propre vécu; elle représente une rupture par rapport à notre attitude spontanée ; elle est à réactiver à chaque étape avec une qualité à chaque fois différenciée ; la deuxième est une phase de redirection, i.e. de conversion de l'attention de «l'extérieur » à «l'intérieur »; la troisième se définit comme un lâcher-prise, qui correspond à un espace d'accueil et d'ouverture.

25. Je me situe ici sur le plan de la critique: concernant l'argumentation empirique/ expérientielle de ce point, je renvoie à l'article de C. Petitmengin dans ce volume, intitulé : « La dynamique pré-réfléchie de l'expérience vécue ».

26. À propos de cette description de la dynamique de l'acte de la prise de conscience, nous renvoyons ici à l'Introduction de N. Depraz, F.J. Varela et P. Vermersch, On becoming aware: the pragmatics of experiencing, Amsterdam, Benjamins Press, 2003. Le texte français est sous presse chez Zeta Books sous le titre A l'épreuve de l'expérience. Pour une pratique phénoménologique. Pour une première élaboration de ce point, cf. «La réduction à l'épreuve de l'expérience », Études phénoménologiques, 2000; en anglais "The Gesture of Awareness» in: M. Velmans ed., Amsterdam, Benjamins Press, 2000, et en allemand: "Die phänomenologische "Epochè" als Praxis » in : Epochè und Reduktion. Formen und Praxis der Reduktion in Phänomenologie (R. Kühn \& M. Staudigl éd.), Königshausen \& Neumann, Orbis Phaenomenologicus, Würzburg, 2003, p. 213-229. 


\section{L'attention}

Aussi, la désignation de ce processus par le préfixe "pré », comme en témoigne l'abondance des termes qui cherchent à le nommer ainsi, mais seulement, au fond, par défaut (de façon générique, on l'a noté initialement: pré-réfléchi, mais aussi, plus spécifiquement: pré-discursif, pré-noétique, anté-prédicatif, pré-verbal, pré-logique ou pré-conceptuel ${ }^{27}$ ) reste verbale. Elle ne dit pas grand-chose du contenu et du mode expérientiel du processus, à savoir, pour commencer, suivant le versant cognitif de l'attention redirectionnelle, de sa composante temporelle, qui reste implicite dans le "pré » de "pré-réfléchi », ou bien, a fortiori, suivant le versant d'ouverture émotionnelle à la réceptivité, de sa composante affective 28 , laquelle ne transparaît quant à elle absolument pas via cette désignation, lors même qu'elle est partie intégrante du processus. Or, ces deux mouvements internes, temporel et affectif, procurent à la dynamique attentionnelle sa densité expérientielle incarnée ${ }^{29}$, ce qui évite $\mathrm{d}^{\prime} \mathrm{y}$ voir, sur le mode kantien de la «condition de possibilité de l'expérience», un état formel inexpérimentable, formulé avec tant d'assurance par le terme malencontreux de « pré-réfléchi ».

Tout en reprenant ici le propos global de l'Introduction de On becoming aware (jusque dans certains de ses contenus et modes d'expression locaux), j'ai pris le parti et la liberté d'en proposer une reformulation qui met en évidence la difficulté du vocabulaire de la réflexion, c'est-à-dire, principalement ici, du vocable "pré-réfléchi », lequel est devenu, à mon sens sur la base d'un malentendu et d'une véritable erreur expérientielle, le nom véhiculaire de cette dimension de la conscience irréductible à la réflexivité alias conscience de soi. C'est pourquoi, j'ai pris le soin de ne pas mobiliser ce vocabulaire dans la description ci-dessus (y compris les expressions connexes mais, à mon sens, sources de confusion d'activité et d'acte réfléchissants), pour privilégier l'expression de "dynamique attentionnelle ». Je livre ainsi, par cette réécriture, une auto-critique du projet de 2003, tout en en réinvestissant les attendus méthodologiques principaux.

27. À propos de ces dénominations insuffisantes, et de la distinction initiale entre conscience pré-réfléchie/préréflexive et conscience réflexive, cf. M. Merleau-Ponty, Phénoménologie de la perception, Paris, Gallimard, 1945, G. Brand, Ich, Welt und Zeit, Den Haag, Martinus Nijhoff, 1955, et les discussions plus récentes dans S. Gallagher, The Inordinance of Time, Evanston, Northwestern Univ. Press, 1998 ; D. Zahavi, Self-Awareness and Alterity, Evanston, Northwestern Univ. Press, 1999 ; et J.L. Bermudes, The Paradox of Self-Awareness, Cambridge, MIT, 1998.

28. A propos de cette composante affective essentielle dans le processus de temporalisation du devenir-conscient, cf. N. Depraz, «Temporalité et affection dans les manuscrits tardifs sur la temporalité de E. Husserl (1929-1936) ", Alter, n², 1994, ainsi que "Can I anticipate myself ? Self-affection and temporality », in Self-awareness, temporality and alterity (D. Zahavi ed.), Dordrecht, Kluwer, 1998 ; cf. par ailleurs E. Husserl, Analysen zur passiven Synthesis, Hua XI, Den Haag, M. Nijhoff, 1966 et P. Ricœur, Philosophie de la volonté I. Le volontaire et l'involontaire, Paris, Seuil, 1950.

29. À propos de ce tressage originaire entre attention, temps et affect, cf. N. Depraz, Comprendre la phénoménologie. Une pratique concrète, Paris, A. Colin, coll. « Cursus », 2006. 


\section{Prendre conscience, devenir-attentif : comment nommer ce processus ? Cécité attentionnelle et synthèse passive}

Dans ce deuxième temps, je vais à présent $m$ 'attacher à rendre compte de cette dynamique attentionnelle. Pour ce faire, je voudrais mettre en évidence un aspect très particulier de l'attention, qui répond à mon sens à une de ses modalités principales d'apparition: je voudrais insister sur sa dimension processuelle. Vous êtes en train de me lire, mais votre attention n'est pas homogène ni constante, elle varie, fluctue sans cesse, connaît des ruptures, vous passez sans cesse d'une micro-phase focalisée à une autre, d'absence (absentmindedness comme disait James), puis à une autre encore de flottement, ou d'ouverture réceptive, voire d'attente, bref, ce n'est en rien un état (avec ses propriétés de stabilité, d'unité, de fixité); j'ai souligné ailleurs son mécanisme de base en termes de «modulation », i.e. sa qualité intrinsèquement variative-transformative, et toujours accompagnante, modifiante voire affectante d'un autre acte ${ }^{30}$.

Je vais m'intéresser à présent à un segment assez ténu de l'attention, qui correspond à sa phase d'apparition initiale (on pourrait parler de sa naissance, de son avènement, ou encore de son émergence, de son engendrement, mais tous ces termes sont lourds de détermination); du fait du moment de l'attention qui $\mathrm{m}$ 'intéresse, je vais être amenée à ré-interroger à cette lumière la relation complexe entre l'attention et la conscience, qui fait l'objet, on l'a montré déjà plus haut, de nombreuses discussions en sciences depuis quelques décennies, mais reste curieusement in-interrogée en phénoménologie, du moins dans le format d'une distinction claire, et quoiqu'elle soit susceptible de fournir des ressources étonnantes en vue de l'élaboration d'une telle distinction; pour pouvoir rendre compte de façon plus précise du processus en jeu, je vais mettre en présence deux mécanismes expérientiels portés par les expressions de "synthèse passive » et " cécité attentionnelle », et situer à partir de là la place de l'attention dans la dynamique de la conscience, sachant que, historiquement, l'attention a été présentée comme une fonction locale et la conscience comme une structure globale.

La thèse que je défends a contrario est que l'attention occupe une place globale et originaire, bref, intégrative, au point que les deux expressions «prendre conscience », « devenir-attentif » renvoient en réalité à un seul et même processus. Cette thèse m'amène à aller à l'encontre de deux options assez souvent disponibles dans la littérature aussi bien philosophique que scientifique : 1) voir dans l'attention une fonction locale et dérivée, assez

30. Cf. N. Depraz (2004), «Where is the Phenomenology of Attention that Husserl intended to perform? A transcendental pragmatic-oriented description of attention» in: Continental Philosophy Review 37 ; Special Issue (A. Steinbock \& N. Depraz éd.) «L'attention » : p. 5-20 ; la traduction française de cet article est parue dans un volume édité par J. Benoist, Husserl (Paris, Cerf, 2008) sous le titre « L'attention comme modulation». 


\section{L'attention}

tardive dans la relation à soi et l'émergence de la conscience, ce qui a pour effet de l'identifier très souvent, à mon sens de façon totalement indue, au mouvement de la réflexion (en philosophie), et ce qui entérine l'idée selon laquelle de nombreux processus perceptifs se déroulent de façon automatique, sans avoir besoin d'attention (en psychologie et en neurosciences) ; 2) faire usage d'adjectifs comme " pré-conscient » et "pré-attentif », qui entérinent l'idée qu'il existe en moi-même une zone expérientielle « sans conscience » et «sans attention", comme s'il y avait quelque chose en moi "avant» la conscience, "avant» l'attention, ce qui à mon sens introduit des biais malheureux et extrêmement dommageables dans l'entente du phénomène en jeu, à savoir une logique binaire (rien/tout ; avant/après) assez abstraite et qui met un mot sur une case expérientiellement vide. Quoi qu'on en pense, et même si on voit dans les mots de simples indicateurs du sens et de l'expérience, ils informent notre façon de penser, et l'expérience elle-même. Bref, mon idée est qu'il n'y a pas de processus pré-conscients à la source de l'attention, pas plus qu'il n'y a de dynamiques pré-attentionnelles qui la précéderaient et dont elle serait l'état subséquent.

Or, pour décrire une telle dynamique intégrative, il se trouve que l'on dispose du côté de la phénoménologie comme des sciences psychologiques et neurologiques de ressources remarquables, non nécessairement symétriques ni homogènes d'ailleurs, mais qui dessinent une cartographie assez fascinante, car les deux niveaux d'analyse sont à même, ici, non pas seulement de se corréler (comme c'est le cas pour la modulation), mais, plus encore, de se générer mutuellement l'un l'autre : la structure de base de cette co-génération se nomme en phénoménologie "synthèse passive » et en sciences inattentional blindness (cécité attentionnelle) : elle s'inscrit dans le cadre d'un mode de conscience situé en deçà de la référence à l'objet et à la relation à un soi unifié, et non nécessairement corporel, même si le corps peut en donner une indication; sur cette base, deux composantes qualifient ce mode de conscience passif ou aveugle, c'est selon, selon le temps et selon l'émotion: la composante "temporelle», que l'on appréhende en phénoménologie dans les termes du "présent vivant », cette dynamique de présence qui inclut intrinsèquement et est ouverte sur le tout juste passé (dit « rétentionnel ») et sur le tout juste à venir (dit «protentionnel»), et en psychologie dans le cadre émergentiste de l'implicit learning (apprentissage implicite); deuxièmement, la composante émotionnelle, qui se décline en phénoménologie en termes de "plaisir», $d^{\prime}$ " affection » et $d^{\prime}$ " affect», et se formule dans les neurosciences sous l'expression d'une «facilitation », d'une «potentiation » ou d'un « enhancement » de l'attention par l'émotion.

Je m'en tiendrai aujourd'hui à la mise en relation des descriptions en phénoménologie et en sciences de la synthèse passive et de la cécité attentionnelle, pour vérifier les zones de recoupement et les points d'irréductibilité, et tenter de valider sur cette base l'hypothèse d'une portée intégrative de l'attention, c'est-à-dire de la prégnance du motif de l'attention 
à la base même de la relation inchoative qui se crée en moi avec le monde, certains de ses objets ou de ses aspects.

La conception de la conscience sur laquelle je fais fond dans cette perspective est globalement "émergente », c'est-à-dire qu'elle n'a pas pour propriété un rapport à l'objet formé comme un vis-à-vis de moi, ni, par conséquent, un soi opérant à titre d'unité même minimale ou immanente ; bref, la conscience coïncide ici avec un devenir-conscient.

\section{A. Le mécanisme en jeu dans la synthèse passive : la genèse affective de l'attention}

«(...) il doit bien déjà y avoir quelque chose de conscient pour le moi, pour qu'il puisse se tourner (sich wenden $z u$ ) en général vers ce quelque chose, et sans conversion attentionnelle (Zuwendung) il n'y a aucune activité en relation avec ce quelque chose (dieses Etwas). La conversion attentionnelle présuppose l'affection, mais, encore une fois, seul quelque chose de conscient peut affecter, à savoir exercer sur le moi un "attrait" plus ou moins grand.

Nous ne souhaitons pas ici mesurer si une telle gradualité de l'affection ne désigne pas simplement une modalité d'un avoir-conscience général, d'un avoir-conscience passif, préalable à une activation possible et, tout d'abord, à la possibilité de la conversion attentionnelle, préalable au commencement de tout "intérêt" du moi, de l'être-là (Dabeisein) auprès de quelque chose et, précisément, de la "mobilisation" à son propos ${ }^{31}$. »

Dans cet extrait, Husserl évoque l'hypothèse selon laquelle un « moi » ne peut être attentif qu'à un «quelque chose » de déjà conscient pour lui. Quel est ce mode de conscience propre au "quelque chose ", qui le relie dès le départ à un moi dont l'attention s'éveille dès lors à lui ? Un type de conscience est en jeu entre le «quelque chose » et le «moi » (en deçà de la distinction entre l'objet et le sujet, et qui n'est pas la conscience explicite ou de soi) : cet « avoir-conscience général » met en mouvement la " conversion attentionnelle ». La modalité de conscience ici évoquée est dite par Husserl " passive », et n'appartient ni au sujet constituant, ni à l'objet constitué, mais à un mode de relation de l'un à l'autre antérieur à leur distinction. Ce mode relationnel de conscience situé en deçà, et de l'objet construit comme vis-àvis, et du sujet égoïque, mode dit " passif », est identifié à partir du motif de l'affection, mais il est dit également générer l'affection elle-même du moi par ce quelque chose déjà « conscient ». Husserl ne caractérise pas plus avant le genre d'avoir-conscience qui se trouve à la base de la genèse affective de l'attention. Dans le deuxième paragraphe, il indique sur un mode plus génétique, mais négatif, comment une telle conscience inclut à titre modal une affection graduelle. En tout état de cause, il s'agit d'une modalité de conscience préalable à tout intérêt et toute activité du moi.

31. Hиа XIV, Appendice II, juin 1921, p. 44. 


\section{L'attention}

On a en fait la dynamique graduelle suivante: un quelque chose de «conscient » > une affection > une conversion attentionnelle > un intérêt > un être-là du moi auprès du quelque chose>un regard porté par le sujet égoïque sur l'objet constitué comme unité de sens. La conversion attentionnelle est la phase-charnière de ce processus, et mon hypothèse est que l'ensemble de ce processus dans ses six différentes phases décrit une dynamique où l'attention joue à plein dès la première phase du "quelque chose de conscient $»$.

Un « quelque chose » (Etwas) m'affecte (ces paroles du serveur au café où je suis en train d'écrire). Ce bruit ne m'affecterait pas s'il n'était déjà là pour moi, selon une structure anticipative qui caractérise à la base la structure de l'expérience attentionnelle, quel que soit mon degré de surprise, d'attente voire de préparation ${ }^{32}$. Bref, « au moment où » ces paroles m'affectent, j'ai déjà en moi-même l'espace d'une entente ${ }^{33}$.

Cette modalité affective de conscience qui co-appartient au quelque chose et à moi-même définit le mouvement élémentaire de l'attention. Husserl parle de "genèse » de l'attention pour identifier un processus qui ne relève pas de la "saisie» (explicite, réfléchie). Aussi est-il à ce stade difficile de savoir si ce processus est linéaire c'est-à-dire s'il se construit sur le mode avant/après, ou s'il ne s'agit que d'une expression conventionnelle qui ne correspond pas à la réalité d'un processus fondamentalement circulaire. Si l'on se centre sur la phase d'amorçage, de "naissance» du processus attentionnel, le modèle génétique de Husserl ${ }^{34}$ comprend trois microétapes: (1) une étape initiale où apparaît la structure de tout ce qui pourra venir à l'éveil de la conscience, que le phénoménologue décrit comme un « champ de donation passive" ou encore une "synthèse passive " cooriginairement temporelle ( $c^{\prime}$ est ce que Husserl nomme dans la première citation un «quelque chose de conscient »), (2) un mouvement de gradualité affective où se trouve exercée une stimulation première (ce qu'il nomme l'affection), (3) un phénomène d'ouverture d'attentionnelle qui relève d'une expérience d'éveil du moi (ce qu'il identifie à la «conversion attentionnelle»). En amont de ces trois étapes, il y a la potentialité de l'affection (0), nommée par Husserl la «hylè », ce qui ne m'affecte même pas, ce qui n'est pas même donné passivement, mais correspond à la puissance sensorielle et sédimentée de nos habitus, et que Husserl, depuis sa logique vécue de l'attention, tend à mettre entre parenthèses au nom du risque d'immixtion de contenus sensibles empiristes. En même temps, ces schèmes sensoriels habituels de l'individu (qu'on dirait en neurosciences neurovégétatifs et neuro-proprioceptifs) sont là et jouent un rôle structurel de base

32. Hua XI, Dordrecht, Kluwer, 1966, trad. fr. sous le titre De la synthèse passive, Grenoble, Millon, 1998.

33. J'ai décrit ailleurs (dans Lucidité du corps. De l'empirisme transcendantal en phénoménologie, Dordrecht, Kluwer, 2001), ce mode d'anticipation inhérent à la conscience du terme d'« autoantécédance ».

34. E. Husserl, Expérience et jugement, Paris, PUF, 1991, § 15-17. 
dans la mise en mouvement affective-attentionnelle. En aval, il y a la saisie (4), les saisies explicitantes, puis toutes les saisies correspondant à des objets de plus en plus complexes et abstraits (5).

Ainsi, le processus en jeu correspond à un enchaînement de phases, selon un modèle circulaire où l'on ne raisonne plus selon la logique empirique d'un stimulus objectif externe qui déclencherait de façon linéaire, comme une cause son effet, l'attention du sujet, mais selon une logique expérientielle où la mobilisation affective de l'éveil empiète sur et transgresse la distinction causale entre pôle objectif et pôle subjectif, et correspond davantage à un espace de jeu et de relief interactif. Dans ce cadre, ce qui requiert une caractérisation plus précise, c'est la modalité affective de la relation à ce «quelque chose de conscient», dotée d'une structure de champ, qui révèle en ce dernier des propriétés qui se disent en termes de relief, de contraste et de saillance, lesquelles créent une disposition du moi qui se présente comme une dynamique d'accueil réceptif, sur le mode d'un co-engrenage en boucle du moi en éveil sur le quelque chose l'affectant

$\mathrm{Au} \S 26$, Husserl décrit l'expérience de la synthèse passive à partir de l'expérience de l'association. Le terme et ce qu'il charrie en termes de compréhension de la relation interne à la conscience peut étonner le lecteur familier de la tradition empiriste et de David Hume en particulier, ou encore de la pensée psychologique du siècle précédent. En effet, la loi associative met en relation des faits sur le mode de la coexistence et de la succession, en rejetant toute causalité logique, mais aussi toute générativité expérientielle ; or Husserl, s'il n'entend pas le processus qu'identifie le mécanisme de la synthèse passive comme une causalité linéaire (à l'instar de la physique newtonienne), ne le décrit pas pour autant comme une relation énergétique aléatoire de faits internes (à la suite de la psychanalyse aujourd'hui, par exemple). Ce qu'il recherche à travers l'expérience de l'association, c'est une structure dynamique de la conscience qui 1) se compose de chaînes non linéaires, mais qui 2) opère à partir d'un concept fort de ressemblance construit sur la médiation de ce qu'il nomme un «membre-pont $\mathrm{t}^{35}$ ». Dès le début, cette structure dynamique est décrite comme l'articulation de propriétés en empiètement partiel, i.e. en un fusionnement (Verschmelzung) impliquant un contraste, à savoir ni par la fusion, ni par la séparation (alternative abstraite). L'exemple proposé, élémentaire, est celui du «carré blanc »: je regarde cette feuille blanche, qui se présente d'abord à moi comme une unité homogène continue, puis, je peux voir apparaître des nuances, des qualités différentes de blancheur, autant de phases implicites qui font le continuum de blanc, lesquelles peuvent éventuellement se détacher c'est-à-dire se singulariser. Ce qui est frappant dans cette description, c'est le fait que ce phénomène associatif est identifié indépendamment de l'expérience d'un sujet explicitement conscient de ce 


\section{L'attention}

qu'il fait: l'association peut relever d'une conscience en action (sans qu'il $\mathrm{s}^{\prime}$ agisse d'une « conscience réflexive d'un objet pour soi ${ }^{36}$ »), mais elle peut aussi avoir lieu au sein de mon champ de conscience sans être l'objet d'une attention expresse: «(..) des associations se déroulent aussi sans être remarquées (bemerkt). De même nous ne faisons pas attention (aufmerksam) à bien d'autres choses qui sont dans notre champ de conscience, nous ne faisons pas attention aux connexions associatives ${ }^{37}$. "

Conclusion : il y a attention et attention. Zuwendung répond au mode de conscience affective (processus d'émergence), Aufmerksamkeit à la notation consciente (remarquer, prêter attention). À ces deux formes d'attention répondent aux modes différents de conscience, l'un en acte, lié à une dynamique affective, l'autre explicite, lié à une identification-repérage, l'une plus organique, l'autre plus signitive. Mais, dans les deux cas, il s'agit bien d'attention.

\section{B. Qu'est-ce que la cécité attentionnelle (inattentional blindness)?}

Pour décrire ce phénomène, je m'appuie essentiellement sur l'ouvragepionnier et encore très actuel d'Arien Mack et Irvin Rock, Inattentional

36. Hua XI, p. 193.

37. Hua XI, p. 195. On peut donc résumer de façon synthétique l'analyse proposée dans ce premier mouvement en décrivant les huit phases de la dynamique attentionnelle en phénoménologie à partir de E. Husserl, De la synthèse passive (1918-1926) :

(0) en amont: potentialité de l'affection, nommée par Husserl la «hylè » (matière/matériau), ce qui ne m'affecte même pas, ce qui n'est pas même donné passivement, mais correspond à la puissance sensorielle et sédimentée de nos habitus, et que Husserl tend à mettre entre parenthèses au nom du risque d'immixtion de contenus sensibles empiristes dans sa logique vécue de l'attention. En même temps, ces schèmes sensoriels habituels de l'individu (qu'on dirait en neurosciences neuro-végétatifs et neuro-proprioceptifs) sont là et jouent un rôle structurel de base dans la mise en mouvement affectivo-attentionnelle

(1) quelque chose de "conscient» (etwas bewußt) (1921)// une étape initiale où apparaît la structure de tout ce qui pourra venir à l'éveil de la conscience, que le phénoménologue décrit comme un « champ de donation passive » ou encore une « synthèse passive » co-originairement temporelle (1918-26)

(2) affection (Affektion) (1921)// un mouvement de gradualité affective où se trouve exercée une stimulation première (1918-26)

(3) conversion attentionnelle (Zuwendung) (1921)// un phénomène d'ouverture qui relève d'une expérience d'éveil (Weckung) du moi (1918-26)

(4) intérêt (Interesse) // Aufmerksamkeit (notation, marquage qui requiert une conscience explicite)

(5) un être-là du moi auprès du quelque chose (Dabeisein) : relation entre les deux pôles en formation

(6) regard porté par le sujet égoïque sur l'objet constitué comme unité de sens (Umkehrung des Blickes : réduction et intentionnalité)

(7) En aval, il y a la saisie, les saisies explicitantes, puis toutes les saisies correspondant à des objets de plus en plus complexes et abstraits.

Je me permets également de renvoyer à la typologie dynamique proposée par A. Steinbock parallèlement dans l'article dans le présent volume : «Exemplarité, émotions et attention ». 
Blindness (MIT, 1998). Les deux auteurs font état pour commencer de ces nombreux moments dans notre vie quotidienne où nous regardons sans voir (seeing without looking) : je suis en train de conduire et je rate le feu que je n'ai pas vu rouge (absorbée que je suis par la conversation que je viens d'avoir avec un collègue) ; inversement, comme cela fut noté par James, une attente intense peut me conduire à voir ou à entendre quelque chose qui n'existe pas: j'entends des pas ou je crois voir la personne que j'attends depuis longtemps avec impatience, mais ce n'est pas elle. Dans un cas, il y a une activité de perception visuelle mais pas d'attention portée dessus, et l'objet est alors non vu parce que non-attended, dans l'autre, mon attention est tellement intense qu'elle crée des perceptions fictives, il y a donc attention sans perception effective.

Je vais m'intéresser au premier cas de figure, nommé également functional blindness ou inattentional blindness, à savoir une cécité de l'attention qui laisse perdurer une activité perceptive. Ce cas de figure va à l'encontre de l'idée très répandue dans la psychologie naissante (Wundt) et dans la philosophie classique (de Descartes à Fichte), selon laquelle l'attention serait simplement une fonction de mise en relief d'une perception déjà présente, ou encore, pour employer l'image souvent utilisée en psychologie, un « projecteur » qui éclaire un objet déjà donné. J'ai mis en question ailleurs une telle conception en mettant au jour le mécanisme structurellement modulatoire de l'attention. Pourtant, les auteurs cités plus haut, sur la base d'expérimentations que je ne pourrai retracer ici précisément, s'inscrivent en faux contre cette idée aujourd'hui très développée d'une activité perceptive sans attention, énoncée en termes de perception pré-attentive ou d'apprentissage implicite. Ils font l'hypothèse contraire selon laquelle il n'y a pas de perception sans attention, sans pour autant revenir à l'hypothèse régressive de l'attention-projecteur ${ }^{38}$, car ils accordent à l'attention un rôle structurant de l'activité perceptive dès son émergence, et non une fonction externe de surplomb. C'est accorder à celle-ci une place centrale et intégrative, et refuser aussi bien l'attention projecteur que la «perception pré-attentive », qui va de pair avec l'idée de processus inconscients et automatiques.

Je vais donc d'abord restituer l'hypothèse standard, puis faire apparaître l'intérêt de l'hypothèse contraire défendue par Mack et Rock. Dans le cadre

38. On peut résumer synthétiquement les positions scientifiques en présence de la façon suivante: A. l'attention-fonction (statique): deux positions antinomiques. 1. L'attentionprojecteur : une fonction qui surplombe les activités conscientes du sujet et les met en relief en les éclairant (Descartes, Wundt, Schütz, Merleau-Ponty sur un mode critique) vs. 2. L'attentionmodulation: une fonction qui transforme les activités du sujet en les faisant variant intrinsèquement: accompagne et favorise l'activité consciente (Husserl, § 92 Idées directrices I ; en sciences, J. Lecas, L'attention visuelle : de la conscience aux neurosciences, Bruxelles, Mardaga, 1992 ; M. Corbetta and G.L. Shulman, «Imaging Expectations and Attentional Modulations in the Human Brain»; in: J. Braun, Ch. Koch and J.L. Davis eds., Visual attention and cortical circuits, Bradfort Book, MIT Press, 2001.), à quoi s'oppose l'attention-dynamique, détaillée plus loin. 


\section{L'attention}

de l'attention-dynamique, on peut ainsi discerner deux positions en discussion, relativement au sens à accorder à l'expression «inattentional blindness ».

\section{La pré-attention : une perception sans attention}

La «pré-attention » correspond à un processus où la cécité de l'attention laisse agir des perceptions qui relèvent d'un «apprentissage implicite ${ }^{39}$ ». Ici, implicite équivaut à «non conscient» au sens de "pré-conscient », et inattentional blindness peut se traduire comme une "cécité de l'attention". Pour illustrer cette hypothèse, je vais reprendre un exemple référentiel, souvent invoqué, issu de l'article de D.J. Simons \& C.F. Chabris : ${ }^{40}$ on montre un match de basketball en vidéo à des sujets. Une équipe est vêtue de T-shirts blancs et l'autre de T-shirts noirs. Les sujets doivent surveiller l'équipe des blancs et compter les échanges de balles. Pendant le film apparaît soudain une femme déguisée en gorille; elle regarde la caméra et se donne des coups sur la poitrine. 50 \% des sujets ne remarquent pas cette présence pourtant insolite. À la question de savoir s'ils ont remarqué quelque chose d'improbable, comme un gorille, ils répondent «non ». Visiblement, l'orientation de l'attention sur un objet déterminé les a rendus aveugles à l'objet du test, auquel ils n'ont accordé aucune attention: autrement dit, les spectateurs, qui prêtent leur attention à une série d'événements peuvent manquer un autre événement, même si cet événement a lieu directement sous leurs yeux.

$C^{\prime}$ est ce que les neuroscientifiques nomment aujourd'hui une perception « subliminale », ou encore automatique, c'est-à-dire opérant à mon insu. $\mathrm{Au}$ fond ce cas de figure entérine l'alliance implicite entre attention et conscience, et entre « pré-attention » et « pré-conscience ».

2. L'attention intégrative à la source du processus perceptif

À cela s'oppose la conception défendue, sur la base de protocoles expérimentaux à chaque fois « uniques » (c'est leur originalité), par Mack et Rock $^{41}$. On a affaire alors à une forme d'attention qui a un caractère intégral ou intégratif, comme l'on voudra: toute activité perceptive consciente suppose une forme d'attention; la dynamique attentionnelle place ainsi l'attention à l'amorçage même de la relation inchoative au quelque chose. Du coup, l'expression « inattentional blindness » doit se comprendre comme une attention à l'œuvre à même la cécité perceptive.

Or, cette hypothèse va à rebours de l'idée-standard, défendue par les piliers de l'attention en psychologie cognitive, Ulrich Neisser (1967) et Anne Treisman (1982), selon laquelle l'attention est directionnelle et correspond à

39. Cf. sur ce point, L. Jimenez éd. Attention and Implicit Learning, Amsterdam/Boston, Benjamins Press, Advances in Consciousness Research, 2002.

40. D.J. Simons \& C.F. Chabris, "Gorillas in our midst: sustained inattentional blindness for dynamic events", Perception vol. 28, 1999, p. 1059-1074.

41. A. Mack et I. Rock, Inattentional Blindness, op. cit. 
une activité subséquente à l'existence préalable d'une chose vers laquelle je me tourne, et qui active mon attention. Cet argument a pour lui la « force de la logique ", mais il s'agit au fond d'une logique empiriste de type causal, atomiste et associationniste, celle-là même que critique Husserl, lequel se rallie à une autre forme de logique, celle de la Gestalt, sur laquelle s'appuient également Mack et Rock. Leur hypothèse auto-organisatrice de l'attention est ainsi en étroite affinité avec l'attentionnalité phénoménologique de Husserl, inhérente au «quelque chose de conscient », qui seul peut amorcer mon éveil et m'attirer.

Un exemple illustre cette hypothèse : une photographie d'un petit déjeuner est présentée à des sujets durant une durée très brève (200 millisecondes), ce qui correspond à une durée inférieure à une saccade oculaire, inférieure à la possibilité d'une recherche oculaire (à un trajet visuel permettant un changement d'objets). Il est demandé aux sujets de décrire la scène avec le maximum de détails. Le résultat est éloquent: la plupart est capable de donner des précisions sur les personnes, leur position, la scène, les objets présents, alors que l'on a par la rapidité de la vision supprimé toute attention explicite liée à une recherche visuelle. Il y a donc à l'œuvre une forme d'attention d'un autre ordre (différente de l'effort et du contrôle), et qui supporte l'activité perceptive ${ }^{42}$.

Dès lors, le «in » de « inattentional » peut être entendu soit comme un privatif, une négation (inattentif, absence d'attention), soit comme une ouverture (le « ad » latin, vers), une intégration. Dans le titre même de leur ouvrage, Mack et Rock font ressortir le terme «attention » (en jaune) sur le blanc («In...al Blindness»), comme pour marquer la présence cruciale de l'attention au cœur du processus, au même moment où ils nous en proposent l'expérience en première personne dans notre activité de lecture du titre de l'ouvrage. Lorsque je lis le titre, je regarde sans voir, prise que je suis par le contenu sémantique et absente à la double coloration, qui me fait voir autre chose. Il y a une attention subconsciente, pourrait-on dire, organique, distincte de l'attention focalisée ici sur la signification ou sur l'identification, qui relève davantage $\mathrm{du}$ mouvement $\mathrm{d}$ 'une prise de conscience, que d'une forme de réflexion.

\section{Conclusion : processus attentionnel et dynamique de la conscience}

Le débat scientifique sur le phénomène assez unique de l'inattentional blindness met en évidence deux interprétations ou modèles de compréhension du processus: lorsque je lis le titre de l'ouvrage, je vois l'expression sémantique et ne fais pas attention au code des couleurs, puis, je le remarque, je m'aperçois de la différence ; si je formule mon expérience ainsi,

42. Op. cit., p. 165 sq. 


\section{L'attention}

l'attention intervient tardivement dans le processus et le premier temps relève d'une activité perceptive dite pré-attentive ; cette interprétation repose sur la dichotomie non/pré-conscient // conscient. L'autre interprétation part du constat d'une activité de lecture intégrée : je lis le titre et mon regard est organiquement attiré par le relief affectif proposé par le contraste entre les deux couleurs, même si cet attrait, justement parce que cet attrait ne relève pas d'une conscience explicite, est doté d'une structure dynamique anticipatrice. L'attention est à l'œuvre dès l'amorçage de la lecture, de pair avec un mode de conscience affective.

La position scientifique du débat pourrait laisser penser que nous avons affaire à un phénomène et à deux interprétations, au fond relatives, et qui tiennent au postulat initial concernant le sens que nous voulons donner à l'attention (précoce, cruciale ou dérivée, locale) et à la conscience (dichotomique ou modale). L'intérêt de l'éclairage proposé par la phénoménologie est de ne pas entériner trop rapidement cette distinction entre le phénomène et le modèle (ce qui risque de reconduire, de façon " méta », le concept d'attention comme projecteur externe), mais de présenter une isomorphie entre l'expérience et le langage qui la porte. Les huit phases descriptives présentées ci-dessus à la note 37 font ressortir la dynamique attentionnelle, l'épousent, et proposent par ailleurs deux qualités d'attention (Zuwendung; Aufmerksamkeit), qui répondent à des moments distincts du processus (organique, signitive), c'est-à-dire aussi à des modes distincts de conscience (affective; notationnelle).

Si l'on prolonge, ce qui reste ici à faire, un tel apport de la phénoménologie par une approche introspective d'auto-explicitation en première personne, on peut accroître la validité de l'hypothèse attentionnelle à la source de tout phénomène proposé par Mack et Rock. On y développerait ainsi, sur la base de descriptions (reports) assez fines déjà indiquées par ces derniers en 1998, une capacité accrue par l'exercice ${ }^{43}$ et le rappel orienté et réitéré des traits du vécu à décrire.

43. A propos de l'importance de l'exercice (training aujourd'hui en anglais), cf. le chapitre $4 \mathrm{du}$ volume 1 de la Psychologie du son de C. Stumpf, traduit ici même. 